\title{
Prática da episiotomia e fatores maternos e neonatais relacionados
}

\author{
Practice of episiotomy and related maternal and neonatal factors
}

Práctica de la episiotomía y factores maternos y neonatales relacionados

Ana Karina Marques Salge ${ }^{1}$, Sara Fleury Lôbo², Karina Machado Siqueira ${ }^{3}$, Renata Calciolari Rossie Silva ${ }^{4}$, Janaína Valadares Guimarães ${ }^{5}$

\section{RESUMO}

A episiotomia trata-se de uma incisão cirúrgica realizada no momento da expulsão do concepto. O objetivo deste estudo foi avaliar o uso da episiotomia e sua associação com as alterações maternas e neonatais em duas maternidades públicas. A população constituiu-se de parturientes submetidas ao parto normal, no período de junho de 2009 a maio de 2010, mediante revisão dos prontuários. Foram analisados 1.129 prontuários. A episiotomia foi utilizada em $57,55 \%$ ( $n=636$ ) de todos os partos normais e em $83,7 \%$ ( $n=325)$ dos partos de primíparas. A associação entre o uso da episiotomia e a primiparidade é estatisticamente significante $(p<0,001)$. Os dados sugerem a associação da episiotomia e aumento do risco de lacerações graves $(p<0,001)$. O emprego da episiotomia mostrou-se semelhante nas duas maternidades. Os resultados deste estudo ratificam o uso indiscriminado da episiotomia e apontam para a necessidade de propor estratégias para reduzir o emprego dessa técnica.

Descritores: Enfermagem Obstétrica; Episiotomia; Parto Normal; Períneo.

\section{ABSTRACT}

Episiotomy consists of a surgical incision performed at the moment the infant is expelled. The purpose of this study was to assess the use of episiotomy and its association with maternal and neonatal factors at two public maternity hospitals. The study population consisted of parturients who experienced natural childbirth in the period between June 2009 and May 2010, through the review of medical records. Analysis included 1,129 medical records. Episiotomy was used in $57.55 \%$ ( $n=636$ ) of all natural childbirths and in $83.7 \%(n=325)$ of the deliveries involving primiparous women. The association between the use of episiotomy and primiparity is statistically significant $(p<0.001)$. Data suggest the association of episiotomy with an increase in the risk of severe lacerations $(p<0.001)$. The use of episiotomy was found to be similar in the two maternity hospitals. The results of this study confirm the indiscriminate use of episiotomy and point out the need for proposal strategies aimed at reducing the use of this technique.

Descriptors: Obstetrical Nursing; Episiotomy; Natural Childbirth; Perineum.

\section{RESUMEN}

La episiotomía es una incisión quirúrgica realizada en el momento de la expulsión del feto. Se objetivó evaluar el uso de la episiotomía y su asociación con las alteraciones maternas y neonatales en dos maternidades públicas. La población se constituyó de parturientas sometidas a parto normal, de junio 2009 a mayo 2010, mediante revisión de historias clínicas. Fueron analizadas 1.129 historias. La episiotomía fue utilizada en el $57.55 \%$ ( $n=636$ ) de los partos normales y en $83,7 \%$ ( $n=325)$ de los partos de primerizas. La asociación entre el uso de la episiotomía y la primiparidad es estadísticamente significativa $(p<0,001)$. Los datos sugieren asociación de la episiotomía y aumento de riesgo de laceraciones graves $(p<0,001)$. El empleo de la episiotomía se mostró semejante en ambas maternidades. Los resultados de este estudio ratifican el uso indiscriminado de la episiotomía y expresan la necesidad de proponer estrategias para reducir el empleo de esta técnica.

Descriptores: Enfermería Obstétrica; Episiotomía; Parto Normal; Perineo.

\footnotetext{
${ }^{1}$ Enfermeira, Doutora em Patologia. Professora Adjunto da Faculdade de Enfermagem (FEN) da Universidade Federal de Goiás (UFG). Goiânia, GO, Brasil. E-mail:anakarina@fen.ufg.br.

${ }^{2}$ Enfermeira, Mestre em Enfermagem. Goiânia, GO, Brasil. E-mail: sarafleury1@yahoo.com.br.

${ }^{3}$ Enfermeira, Mestre em Enfermagem. Discente do Programa de Pós-Graduação em Enfermagem da FEN/UFG, nível Doutorado. Professora Assistente da FEN/UFG. Goiânia, GO, Brasil. E-mail:karinams.fen@gmail.com.

${ }^{4}$ Fisioterapeuta, Doutora em Ciências da Saúde. Professora do Departamento de Patologia da Universidade do Oeste Paulista. Presidente Prudente, SP, Brasil. E-mail: renata@unioeste.br.

${ }^{5}$ Enfermeira, Doutora em Patologia. Professora Adjunto FEN/UFG. Goiânia, GO, Brasil. E-mail: valadaresjanaina@gmail.com.
} 


\section{INTRODUÇÃO}

A episiotomia é uma incisão cirúrgica na região da vulva, realizada no momento da expulsão do concepto(1). Os benefícios, descritos na literatura, incluem a prevenção do trauma perineal grave e das lesões desnecessárias do pólo cefálico do recém-nascido (RN), melhoria da futura função sexual e ainda facilitação do reparo, pois substitui uma laceração irregular por uma incisão limpa e regular ${ }^{(1)}$.

Trata-se de um dos procedimentos mais comuns em obstetrícia, sendo superado apenas pelo corte e pinçamento do cordão umbilical. É praticado em $60 \%$ dos partos normais, com frequência de até $90 \%$ em primíparas ${ }^{(2)}$.

Nos últimos anos, a literatura tem demonstrado que o uso da episiotomia de rotina não deve ser incentivado, abrindo a discussão sobre os benefícios e riscos deste procedimento(3). Dentre os riscos e prejuízos estão a perda sanguínea, prolapso, retocele, cistocele, incontinência urinária, dispareunia, piora da função sexual e aumento dos índices de infecção. Soma-se a isto o fato de que a episiotomia não previne lacerações graves, de terceiro e quarto graus ${ }^{(4-5)}$.

Altos índices de episiotomia contrariam as diretrizes preconizadas pela Organização Mundial da Saúde (OMS) que considera a episiotomia como prática frequentemente utilizada de modo inadequado e que deve ter seu uso limitado(6).

Estudos apontam que esta prática é menos comum quando há a preocupação com a humanização ao atendimento à parturiente ${ }^{(7-9)}$. $O$ princípio da humanização no campo obstétrico hospitalar está centrado em uma assistência menos intervencionista. São utilizados métodos não invasivos que contribuem de maneira eficiente para a progressão do parto e respeitam os direitos sexuais e reprodutivos da mulher ${ }^{(10)}$.

A assistência humanizada ao parto é muito discutida pelos enfermeiros obstetras. Há uma preocupação em instituir tecnologias que auxiliem na fisiologia do parto e preservem a integridade corporal, evitando intervenções desnecessárias como a episiotomia ${ }^{(11-13)}$.

No Brasil, o emprego da técnica pelo profissional enfermeiro foi regulamentado pela Lei Federal $n^{\circ} 7.498$ de 1.986 e pelo Decreto $n^{\circ} 94.406$ de 1.987 , que respalda a realização de episiotomia e episiorrafia pelo profissional enfermeiro com formação em obstetrícia. A inclusão do procedimento parto normal sem distócia realizado por enfermeiro na tabela do Sistema de Informações Hospitalares (SIH) do Sistema Único de Saúde (SUS), regulamentada pela Portaria Ministerial $n$. 2815/98, viabilizou a emissão de Autorização de Internação Hospitalar (AIH), incentivando a realização de partos normais por enfermeiros obstetras.

No Brasil, além do uso indiscriminado da episiotomia, em alguns casos, os altos índices de cesarianas são justificados erroneamente, como forma de prevenção de danos provocados pelo uso dessa técnica nos partos normais. Por esse motivo, a discussão sobre riscos, benefícios e fatores relacionados ao uso da episiotomia é extremamente válida. Portanto, o objetivo desse estudo foi avaliar a prática da episiotomia em duas maternidades públicas e identificar os possíveis fatores maternos e neonatais relacionados em sua ocorrência.

\section{METODOLOGIA}

Trata-se de um estudo retrospectivo de corte transversal. A população constituiu-se de parturientes submetidas ao parto normal em duas maternidades públicas na cidade de Goiânia, Goiás, no período de junho de 2009 a maio de 2010. O referido período não corresponde a fase de coleta de dados, mas ao período de seleção (recorte) da demanda que está sendo analisada, mediante revisão dos prontuários.

A maternidade A pertence a um Hospital Público Universitário do município de Goiânia, Goiás, o qual, desde o ano de 2002, integra o grupo dos 188 hospitais que formam a Rede de Hospitais sentinela em todo o país junto à Agência Nacional de Vigilância Sanitária (ANVISA). Esta maternidade atende, prioritariamente, gestantes com diagnóstico de gravidez de alto risco atendidas pelo Sistema Único de Saúde (SUS). No período de um ano, entre junho de 2009 e maio de 2010 foram realizados 325 partos normais na instituição, entretanto devido ao critério de exclusão (óbito fetal), foram estudados 282 prontuários (86,76\%).

A Maternidade B é uma unidade da Secretaria de Estado da Saúde de Goiás (SES/GO), que atende majoritariamente uma clientela de baixo nível sócioeconômico, usuários do Sistema Único de Saúde. Atualmente equipada com 38 leitos e realizando cerca de 150 partos por mês, no período estudado registrou 887 partos normais. Destes, 850 prontuários foram analisados. Os demais não entraram no estudo devido 
aos critérios de inclusão.

No período estudado foram registrados 19.448 partos em mulheres residentes em Goiânia. Destes 6.418 foram normais e 12.989 partos por cesarianas e 41 ignorados. Desta maneira, estas maternidades representam 17,6 \% dos partos normais ocorridos em Goiânia neste período. Nesse período, todos os partos foram realizados exclusivamente por profissionais médicos, pois não havia enfermeiros obstetras inseridos diretamente na assistência ao parto normal sem distócia.

Foram incluídas no estudo parturientes que foram submetidas ao parto normal, dando a luz a feto vivo, no período estudado.

A fonte de informação para esse estudo foi composta pelo prontuário médico de cada paciente, de onde foram coletados e transcritos em formulário próprio dados sócio-demográficos (idade, procedência, escolaridade, profissão); dados obstétricos (número de gestações, número de partos, número de abortos, realização de pré-natal, idade gestacional em que ocorreu o parto). Também foram coletados dados referentes ao emprego da episiotomia (uso e tipo de episiotomia utilizada) e presença de lacerações de acordo com o grau. As doenças de base maternas e neonatais, as possíveis intercorrências obstétricas maternas e fetais durante o período expulsivo e pósparto, as medidas antropométricas neonatais (peso ao nascer, estatura, perímetro cefálico, perímetro torácico), Índice de Apgar no $1^{\circ}$ e $5^{\circ}$ minutos também foram pesquisados.

Para a análise estatística foi elaborada uma planilha eletrônica. As informações foram analisadas por meio do programa eletrônico Sigma Stat ${ }^{\circledR}$, versão 2.0. As variáveis categóricas são apresentadas em seus valores absolutos (n) e relativos (porcentuais). As proporções foram comparadas pelo teste de correção de Yates ou teste do Qui-Quadrado $\left(\chi^{2}\right)$ e teste exato de Fisher. Foram consideradas estatisticamente significantes as diferenças em que $\mathrm{p}$ foi menor que $5 \%$.

O projeto foi aprovado pelo Comitê de Ética em Pesquisa Médica Humana e Animal do Hospital das Clínicas da Universidade Federal de Goiás, sob o número de protocolo 131/08. Todas as etapas da pesquisa respeitaram as recomendações propostas pelo Conselho Nacional de Saúde, Resolução 196/96(14).

\section{RESULTADOS}

Foram analisados 1.129 prontuários de parturientes submetidas ao parto normal, a episiotomia foi utilizada em 636 (57,55\%) de todos os partos normais e em 325 $(83,7 \%)$ dos partos de primíparas. Considerando o número total de parturientes, a média ( \pm DP) da idade foi

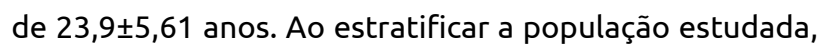
$193(17,1 \%)$ parturientes apresentaram idade igual ou inferior a 18 anos; 891 (78,9\%) de 19 a 35 anos e 44 $(3,9 \%)$ possuíam idade superior a 35 anos.

A média das idades foi semelhante nas duas maternidades, sendo de $23,9 \pm 6,2$ anos na maternidade $A$ e de 24,0 $\pm 5,6$ anos na maternidade $B$.

O número de primigestas foi semelhante ao de multigestas, somando 393 (34,8\%) e 392 (34,7\%), respectivamente. A maternidade A atendeu 112 (40,1\%) primigestas e $85(30,4 \%)$ multigestas, enquanto que na maternidade $\mathrm{B}, 281(33,0 \%)$ das mulheres atendidas eram primigestas e 307 (36,1\%) já haviam engravidado três ou mais vezes.

A média da idade gestacional (IG) em que ocorreu o parto na maternidade $A$ foi de $37,5 \pm 3,6$ semanas. Já a maternidade $B$ apresentou média de IG de 38,4 $\pm 3,6$ semanas.

Houve predomínio do parto a termo em 545 (48,3\%) casos. Foram identificados 311 (27,6\%) partos pós-termo e $258(23,0 \%)$ partos pré-termos. Dentre os prematuros, 24 (9,30\%) eram prematuros extremos, 128 (49,6\%) prematuros moderados e 106 (41,0\%) apresentaram prematuridade limítrofe. Como observado na Tabela 1, a maternidade A apresentou, proporcionalmente, um número maior de partos pré-termo (10,4\%). Provavelmente este achado está relacionado ao perfil desta instituição. 
Tabela 1: Número de partos de acordo com a idade gestacional de parturientes submetidas ao parto normal em duas maternidades públicas no período de junho de 2009 a maio de 2010. Goiânia, GO, 2010.

\begin{tabular}{cccc}
\hline Idade Gestacional & $\begin{array}{c}\text { Maternidade A } \\
\mathbf{n}(\%)\end{array}$ & $\begin{array}{c}\text { Maternidade B } \\
\mathbf{n}(\%)\end{array}$ & $\begin{array}{c}\text { Total } \\
\mathbf{n}(\%)\end{array}$ \\
\hline Pré-termo & $104(37,3)$ & $156(18,4)$ & $260(23,0)$ \\
A termo & $104(37,3)$ & $442(52,0)$ & $546(48,3)$ \\
Pós-termo & $69(24,7)$ & $242(28,5)$ & $311(27,6)$ \\
SI* & $02(0,7)$ & $10(1,1)$ & $12(1,1)$ \\
\hline Total & $\mathbf{2 7 9 ( 1 0 0 )}$ & $\mathbf{8 5 0 ( 1 0 0 )}$ & $\mathbf{1 1 2 9 ( 1 0 0 )}$ \\
\hline
\end{tabular}

SI: sem informação; n: número.

Em relação às comorbidades maternas, observou-se $121(10,7 \%)$ casos em que foram relatadas alterações maternas. O grupo de doença de base que se fez mais presente foi o das doenças infecciosas, em ambos os serviços, representando $50,4 \%$ (61) dos casos. Não foi observada relação estatisticamente significante também entre as alterações maternas e o uso da episiotomia $\left(\chi^{2}=1,649 ; p=0,199\right)$.

$\mathrm{O}$ peso médio dos $\mathrm{RN}$ foi de 3.105,48 $\pm 543,39$ gramas. Quando analisados separadamente os dois serviços, percebeu-se que na maternidade $A$ o peso médio dos RN foi de 2.857,36 $\pm 726,50$ gramas, menor do que 0 identificado na maternidade $B$, de $3.105,48 \pm 543,39$. No grupo em que a episiotomia foi praticada, a média do peso foi de 3.110,65 gramas; e
3.091,00 no grupo em que não foi realizado o procedimento.

A média do índice de Apgar no primeiro minuto foi de 8,07 e de 9,41 no quinto minuto. Quando relacionados ao uso da epsiotomia temos uma média de 7,99 do Apgar de $1^{\circ}$ minuto e 9,38 no $5^{\circ}$ minuto nos casos em que este procedimento foi realizado e de 8,18 e 9,44 , no $1^{\circ}$ e $5^{\circ}$ minuto respectivamente, nos casos em que a episiotomia não foi feita. A alteração neonatal mais frequente em ambos os serviços analisados foi a prematuridade (37,2\%). Não foi observada relação estatisticamente significante também entre as alterações fetais/neonatais e o uso da episiotomia $\left(p=0,622 \chi^{2}=0,244\right)$ (Tabela 2).

Tabela 2: Características antropométricas e índice de Apgar no $1^{\circ}$ e $5^{\circ}$ minuto dos recém-nascidos de parturientes submetidas ao parto normal em duas maternidades públicas no período de junho de 2009 a maio de 2010. Goiânia, GO, 2010.

\begin{tabular}{|c|c|c|c|c|}
\hline \multirow{3}{*}{ Dados RN } & \multicolumn{2}{|c|}{ Matenidade A } & \multicolumn{2}{|c|}{ Maternidade B } \\
\hline & Com episiotomia & Sem episiotomia & Com episiotomia & Sem episiotomia \\
\hline & \multicolumn{2}{|c|}{ Média } & \multicolumn{2}{|c|}{ Média } \\
\hline Perímetro cefálico & $33,5 \mathrm{~cm}$ & $33,3 \mathrm{~cm}$ & $33,4 \mathrm{~cm}$ & $35,0 \mathrm{~cm}$ \\
\hline Perímetro torácico & $31,9 \mathrm{~cm}$ & $31,8 \mathrm{~cm}$ & $32,5 \mathrm{~cm}$ & $33,5 \mathrm{~cm}$ \\
\hline Peso & و $2.889,3$ & $2.816 \mathrm{~g}$ & g.164,72 & $3.171,20 \mathrm{~g}$ \\
\hline Estatura & $50,1 \mathrm{~cm}$ & $49,5 \mathrm{~cm}$ & $48,7 \mathrm{~cm}$ & $48,8 \mathrm{~cm}$ \\
\hline Apgar $1 \mathrm{~min}$ & 7,3 & 7,3 & 8,1 & 8,4 \\
\hline Apgar 5 min & 8,8 & 8,8 & 9,5 & 9,6 \\
\hline
\end{tabular}

RN: recém-nascido; cm: centímetro; g: grama; min: minuto.

O estudo revelou que mais da metade, 636 (57,5\%), das mulheres foram submetidas à episiotomia. Esta prática ocorreu numa frequência semelhante nas duas maternidades, sendo observados 151 (54,5\%) na maternidade A e 485 (58,5\%) casos na maternidade B. A episiotomia médio-lateral prevaleceu como escolha na maior parte dos casos, considerando os informados, 415 (96,7\%). Também foram relatados 13 (3,0\%) casos de episiotomia mediana. A descrição "posterior direita" também foi utilizada em um caso $(0,2 \%)$. Houve associação estatisticamente significante $\left(\chi^{2}=31,623\right.$; $\mathrm{p}<0,001)$ entre a idade materna e a prática da episiotomia.

Quando analisada a relação entre a paridade e o uso da episiotomia, observou-se que este procedimento foi mais prevalente nas primíparas, apresentando diferença estatística entre primiparidade e a realização da episiotomia $\quad\left(\chi^{2}=202,080 ; \quad p=<0,001\right) ;$ quando comparadas as mulheres com partos anteriores.

Considerando o total de prontuários analisados, 
houve referência à 250 (22,5\%) casos de laceração. Destas, 82 (32,1\%) ocorreram em parturientes da maternidade A e $173(67,8 \%)$ na maternidade B. A maternidade $B$ apresentou um maior número de lacerações, porém também apresentou maior número de partos, assim, proporcionalmente ao número de partos realizados, a maternidade $A$ apresentou $29,3 \%$ de partos com laceração e a maternidade B 20,3\%.

A maior parte dos casos de laceração informados, 98 $(77,8 \%)$, ocorreu em mulheres que não foram submetidas à episiotomia. Entretanto, neste grupo, considerando os casos informados, prevaleceram lacerações de $1^{\circ}$ e $2^{\circ}$ graus; $n=89$ (90,8\%). Os casos de lacerações graves, de $3^{\circ}$ ou $4^{\circ}$ graus foram relatados em nove $(9,2 \%)$ casos do grupo de não submetidas à episiotomia, cuja informação foi relatada em prontuário. Nos casos em que houve episiotomia, 13 (46,4\%) das lacerações informadas (28) foram de $3^{\circ}$ ou $4^{\circ}$ graus.

Houve diferença estatisticamente significante $\left(\chi^{2}=126,983 ; p<0,001\right)$ em relação ao uso da episiotomia e a incidência de lacerações. Neste caso, quando a episiotomia não foi realizada o número de lacerações foi maior. Já no grupo de mulheres em que a episiotomia foi realizada (636 casos), 51 (8,0\%) apresentaram lacerações. Destas foram relatadas $13(25,4 \%)$ casos de lacerações de $3^{\circ}$ e $4^{\circ}$ graus.

Assim, foi possível observar uma diferença estatisticamente significante $\left(\chi^{2}=20,012 ; p<0,001\right)$ em relação à prática da episiotomia e risco para lacerações de $3^{\circ}$ ou $4^{\circ}$ graus. Desta maneira, a maior parte das lacerações graves foi encontrada nos casos em que a episiotomia foi praticada.

\section{DISCUSSÃO}

A população foi composta, em sua maioria, por mulheres com idade média de $23,9 \pm 5,6$ anos. A média de idade descrita na literatura, em trabalhos relacionados à parturição, tem variado de acordo com o contexto em que o estudo é realizado(15).

A frequência com que a episiotomia foi praticada nas parturientes foi inferior ao valor encontrado em um estudo realizado na América Latina $(92,3 \%)^{(16)}$ e superior a encontrada em outros estudos nacionais $(37,8 \%$ e de $29,1 \%)^{(17-18)}$. Estudos realizados em instituições em que o enfermeiro obstetra assiste ao parto normal demonstram que a taxa de episiotomia nestes locais é bem inferior daquelas em que apenas os profissionais médicos atuam nesta assistência. Em uma maternidade pública do Rio de Janeiro foi detectada uma taxa de $11,2 \%$ referente ao uso da episiotomia em relação ao total de partos acompanhados em ano por enfermeiros obstetras ${ }^{(12)}$. Em outro estudo realizado em São Paulo esse valor foi de $25,7 \%{ }^{(9)}$.

Alguns autores sugerem que a episiotomia seja realizada entre $10 \%$ a $30 \%$ dos partos normais, embora o Ministério da Saúde não tenha determinado uma taxa de referência para esta prática ${ }^{(19)}$. Os dados encontrados em nosso estudo ultrapassam esta estimativa e apresentam índices superiores aos sugeridos pela OMS.

Em nosso estudo, a primiparidade também foi considerada como um fator predisponente para a realização da episitomia. Alguns autores atribuem à alta prevalência da episiotomia em primíparas devido à diminuição da elasticidade dos músculos perineais, propiciando uma maior rigidez à região no momento impulsivo do parto. Enquanto que, o parto normal, levaria a uma maior distensão do períneo nos partos futuros e consequentemente diminuiria a necessidade de uma nova episiotomia(20).

A faixa etária inferior a 19 anos foi relacionada à maior incidência desta prática, apresentando relação estatisticamente significante $(p<0,001)$. O emprego deste procedimento em mulheres jovens pode ser justificado devido a pelve materna não ter atingido o padrão adulto(21). Em um estudo que busca avaliar o desfecho da gravidez nas adolescentes, outros fatores como paridade, uso de fórceps e macrossomia são mencionados e devem ser considerados ${ }^{(22)}$.

Quanto analisado o peso do RN, não foi encontrada associação com o uso da episiotomia. Em um estudo correlato, 1.576 partos vaginais foram acompanhados e a macrossomia (peso fetal superior a $4.000 \mathrm{~g}$ ) foi detectada como fator de risco para uma episiotomia. Além da macrossomia, o segundo estágio do parto prolongado e o uso da analgesia peridural foram considerados fatores de risco para realização da episiotomia(23).

Foi encontrada uma relação estatisticamente significante entre o uso da episiotomia e o maior risco de lacerações graves, com lesão de esfíncter anal. Em estudos realizados com mulheres primíparas, os resultados apontam redução de laceração de terceiro grau nas mulheres do grupo de episiotomia seletiva, quando comparado com o uso rotineiro. Esses estudos 
referem ainda maior ocorrência de lacerações de $1^{\circ}$ e $2^{\circ}$ graus (periuretral, pequenos lábios e lacerações vaginais superficiais) no grupo que realizou episiotomia de maneira seletiva(24-25). Foi observado que as lacerações de $2^{\circ}$ grau ocorreram em $4,6 \%$ dos partos realizados sem episiotomia e o registro de lesões de $1^{\circ}$ grau corresponderam à $56,6 \%$. Não houve registro de lesões de $3^{\circ}$ e $4^{\circ}$ graus $^{(9)}$.

\section{CONCLUSÃO}

A avaliação da prática da episiotomia realizada por meio da identificação de possíveis fatores maternos e neonatais relacionados mostrou que houve predomínio do uso desse procedimento em primíparas, do parto normal a termo e que a incisão médio-lateral prevaleceu como técnica mais utilizada. Lacerações de $1^{\circ}$ e $2^{\circ}$ graus ocorreram nos casos em que a episiotomia não foi utilizada. As alterações maternas e neonatais não foram estatisticamente significantes quando relacionadas ao uso da episiotomia, sugerindo que os critérios utilizados

\section{REFERÊNCIAS}

1. DeLee JB. The prophylactic fórceps operation. Am. J. Obstet. Gyancol. 1920;1:34-38.

2. Rubio JA. Política selectiva de episiotomía y riesgo de desgarro perineal en um hospital universitário. Rev. Colomb. Obstet. Ginecol. 2005;56(2):116-26.

3. São Bento PAS, Santos RS. Realização da episiotomia nos dias atuais à luz da produção científica: uma revisão. Esc. Anna Nery. 2006;10(3):552-59.

4. Raisanen S, Inen-Julkunen KV, Heinonen S. Need for and consequences of episiotomy in vaginal birth: a critical approach. Midwifery. 2010;26(3):348-56.

5. Geranmayeh M, Rezaei Habibabadi Z, Fallahkish B, Farahani MA, Khakbazan Z, Mehran A. Reducing perineal trauma through perineal massage with vaseline in second stage of labor. Arch Gynecol Obstet. 2012 Jan;285(1):77-81.

6. Organização Mundial de Saúde. Assistência ao Parto Normal: um guia prático. Saúde Materna e Neonatal/ Unidade de Maternidade Segura. Saúde reprodutiva e da Família. Genebra (Suiça): Organização Mundial de Saúde; 1996.

7. Schneck CA, Riesco MLG, Bonadio IC, Diniz CSG, Oliveira SMJV. Resultados maternos e neonatais em centro de parto normal peri-hospitalar e hospital. Rev. Saúde Pública. 2012;46(1):77-86.

8. Silva TF, Costa GAB, Pereira ALF. Cuidados de enfermagem obstétrica no parto normal. Cogitare Enferm. 2011;16(1):82-87.

9. Seibert SL, Barbosa JLS, Santos JMS, Vargens OMC. Medicalização x humanização: o cuidado ao parto na histórica. Rev. Enfem. UERJ. 2005;13:245-51. na prática obstétrica em relação à episiotomia não seguem um padrão determinado.

Este estudo mostra que a realização de procedimentos e intervenções sem uma prévia indicação obstétrica é comum em parturientes, como é o caso da episiotomia de rotina e que precisa ser reavaliada.

Apesar das evidências científicas que questionam o seu uso, observa-se que esta prática ainda é comum nas maternidades estudadas. Estas são maternidades públicas e deveriam seguir as recomendações do MS para realização de partos humanizados. Nota-se ainda a necessidade da maior inserção dos enfermeiros obstetras na assistência direta ao parto normal sem distócia. O seguimento de um modelo menos intervencionista, pautado nos conceitos de humanização, que respeite os direitos femininos e o parto normal fisiológico, podem contribuir de forma positiva na diminuição dos índices de episiotomia e no incremento da atenção ao binômio mãe-filho.

10. Figueiredo GS, Santos TTR, Reis CSC, Mouta RJO, Progianti JM, Vargens OMC. Ocorrência de episiotomia em partos acompanhados por enfermeiros obstetras em ambiente hospitalar. Rev. Enferm. UERJ. 2011;19(2):1815.

11. Progianti JM, Mouta RJO. A enfermeira obstétrica: agente estratégico na implantação de práticas do modelo humanizado em maternidades. Rev. enferm. UERJ. 2009;17(2):165-9.

12. Porfírio AB, Progianti JM, Souza DOM. As práticas humanizadas desenvolvidas por enfermeiras obstétricas na assistência ao parto hospitalar. Rev. Eletr. Enf. [Internet]. 2010 [cited 2012 jan 21];12(2):331-6. Available from:

http://www.fen.ufg.br/revista/v12/n2/v12n2a16.htm.

13. Santos JO, Shimo AKK. Pratica rotineira da episiotomia refletindo a desigualdade de poder entre profissionais de saúde e mulheres. Esc. Anna Nery. 2008;12(4):645-50.

14. Conselho Nacional de Saúde, Ministério da Saúde. Resolução No 196/96 - Normas regulamentadoras de pesquisa envolvendo seres humanos. Brasília (Brasil): Ministério da Saúde; 1996.

15. Koskas M, Caillod AL, Facuconnier A, Bader G. Maternal and neonatal consequences induced by the Frech recommendations for episiotomy practice. Monocentric study about 5409 vaginal deliveries. Gynecol. Obstet. Fertil. 2009; 37(9):697-702. 16. Althabe F, Beizán JM, Bergel E. Episiotomy rates in primiparous women in Latin America: hospital based descriptive study. BMJ. 2002;324:945-46. 
17. Melo Júnior EF. Episiotomia seletiva: frequencia e fatores associados [dissertation]. Recife: Saúde da criança e do adolescente/Universidade Federal de Pernambuco; 2005. 71p.

18. Carvalho CCM, Souza ASR, Moraes Filho OB. Prevalência e fatores associados à prática da episiotomia em maternidade escola do Recife, Pernambuco, Brasil. Rev. Assoc. Med. Bras. 2010;56(3):333-39.

19. Mattar R, Aquino MMA, Mesquita MRS. A prática da episiotomia no Brasil. Rev Bras Ginecol Obstet. 2007;29:1-2.

20. Oliveira SMJV, Miquilini EC. Frequencia e critérios para indicar a episiotomia. Rev. esc. enferm. USP. 2005;39(03):288-95.

21. Phillips C, Monga A. Childbirth and pelvic floor: "the gynecological consequences". BJOG. 2005;5:15-22. 22. Metello J, Torgal M, Viana R, Martins L, Maia M, Cásal $E$ et al. Desfecho da gravidez nas jovens adolescentes. RBGO. 2008;30(12):620-25.

23. Robinson JN, Norwitz ER, Cohen AP, Lieberman E. Predictors of episiotomy use at first spontaneous vaginal delivery. Obstet. Gynecol. 2000;96(2):214-18.

24. Rodriguez A, Arenas EA, Osorio AL, Mendez O, Zuleta $J J$. Selective vs routine midline episiotomy for the prevention of third-or fouth-degree lacerations in nulliparous women. Am. J. Obstet. Gynecol. 2008;198(1)285-94.

25. Foroughipour A, Firuzeh F, Ghahiri A, Norbakhsh V, Heidari T. The effect of perineal control with hands-on and hand-poised methods on perineal trauma and delivery outcome. J Res Med Sci. 2011;16(8):1040-46.

Artigo recebido em 15/04/2012.

Aprovado para publicação em 06/06/2012.

Artigo publicado em 31/12/2012. 